\title{
Prognostic significance of lymphovascular invasion diagnosed by lymphatic endothelium immunostaining in breast cancer patients
}

\author{
KENJI TEZUKA $^{1}$, NAOYOSHI ONODA ${ }^{1,2}$, TSUTOMU TAKASHIMA ${ }^{1}$, KEIICHI TAKAGAKI $^{1}$, \\ TETSURO ISHIKAWA $^{1}$, TOMOKO WAKASA ${ }^{3}$, KENICHI WAKASA ${ }^{3}$ and KOSEI HIRAKAWA ${ }^{1}$ \\ ${ }^{1}$ Department of Surgical Oncology; ${ }^{2}$ Department of Oncology, Institute of Geriatrics and Medical Science, \\ Osaka City University Graduate School of Medicine; ${ }^{3}$ Department of Diagnostic Pathology, \\ Osaka City University Hospital, 1-4-3 Asahi-machi, Abeno-ku, Osaka 545-8585, Japan
}

Received January 4, 2007; Accepted February 9, 2007

\begin{abstract}
Prognostic factors for breast cancer include axillary lymph node status, tumor size, histology, nuclear grade, presence of estrogen and progesterone receptors, HER2/neu status, and mean microvessel density (MVD). In this study, we evaluated the usefulness of a new marker, D2-40, by investigating lymph vascular invasion of the tumor immunohistochemically in 132 patients with breast cancer and compared it with those of well-known prognostic indicators. Positive immunostaining of lymphatic endothelium with D2-40 outlining tumor emboli in the lumen of lymphatics was defined as D2-LVI, and lymphatic invasion following conventional hematoxylin and eosin staining was defined as HE-LVI. Significant correlation was observed between HELVI and D2-LVI $(\mathrm{p}<0.001)$, between lymph node status and HE-LVI ( $\mathrm{p}=0.005)$, and between recurrent status and D2-LVI $(\mathrm{p}=0.008)$ by univariate analysis. Based on multivariate analysis, lymph node status $(\mathrm{p}<0.001, \mathrm{OR}=6.993)$, tumor size $(\mathrm{p}=0.005, \mathrm{OR}=5.504), \mathrm{D} 2-\mathrm{LVI}(\mathrm{p}=0.006, \mathrm{OR}=4.740)$, and MVD ( $\mathrm{p}=0.002, \mathrm{OR}=4.484)$ were independent prognostic factors of disease recurrence. A significant difference in disease-free survival was also found between patients with and without D2-LVI ( $\mathrm{p}=0.0067$ ), but not with or without HELVI. Even in node-positive cases, D2-LVI had prognostic meaning. D2-LVI may play a crucial role for predicting recurrence of breast cancers much more than expected. Our data identifying D2-LVI expression in tumors of patients
\end{abstract}

Correspondence to: Dr Kenji Tezuka, Department of Surgical Oncology, Osaka City University Graduate School of Medicine, 1-4-3 Asahi-machi, Abeno-ku, Osaka 545-8585, Japan

E-mail: tezu@kawachi.zaq.ne.jp

Abbreviations: LVI, lymphovascular invasion; MVD, microvessel density; MVD-L, microvessel density-low; MVD-H, microvessel density-high

Key words: CD-34, D2-40, lymphovascular invasion, prognosis, recurrence with a poor disease-free survival prognosis provides an easier and more accurate prognostic method than identifying HE-LVI.

\section{Introduction}

Factors predicting the prognosis of breast cancer are well established. They include not only conventional clinical factors, such as status of lymph-node metastasis, tumor size, histological type of the tumor, and nuclear grade, but also some molecular biological factors, such as presence of estrogen and progesterone receptors, and expression of HER2/neu molecules in cancer cells. Additionally, at the 2005 St. Gallen consensus meeting, histologically overt lymphatic vessel invasion was recognized as one of the factors that should determine a treatment plan (1).

Indeed, it is well known that lymphatic and blood angiogenesis play important roles in tumor progression. Former studies have suggested that intratumor angiogenesis, measured by micro-blood vessel counts, correlates intimately with the metastatic potential and the prognosis of cancers in many organs including breast cancer. Weider et al (2) first demonstrated the clinical usefulness of quantifying angiogenesis by measuring the microvessels in breast cancer tissue using a specific marker for vascular endothelial cells. Since then, many studies have evaluated microvessel density (MVD) as a prognostic factor for breast cancer. However, evaluation of angiogenesis has not been used as a routine prognostic factor due to the difficulty in standardizing the evaluation method. On the other hand, studies of lymphatic vessels and lymphangiogenesis have been hampered by lack of specific markers, even though breast cancer metastasis occurs more often via the lymphatic system than by hematological spread. Recently, antibodies directed against M2A (D2-40), LYVE-1, Podoplanin, and Prox-1 specifically demarking lymphatic vessels in paraffin-embedded sections have become available (3-5). Several recent studies have suggested the usefulness of these markers to detect lymphatic vessels in breast cancer. According to these limited reports, lympho-vessel involvement or lympho-microvessel density, assessed by immunohistochemistry (IHC) using these markers, may correlate well with lymphatic spread and the prognosis after surgery. Still, there remain some problems to solve, such as the antibody 
and patient selection, method of evaluation and, most importantly, the meaning of adding IHC to the standard H\&E based histological evaluation.

In the present study, we investigated the lymphatic involvement in breast cancer tissues by IHC using the D2-40 monoclonal antibody and compared the results with conventional histological assessment using H\&E stain or blood microvessel counts. Our findings revealed that lymph vessel involvement of the tumor determined specifically with IHC was a strong and independent prognostic factor to predict disease recurrence.

\section{Materials and methods}

Patients. The study included 132 women with breast cancer, diagnosed and treated in the Osaka City University Hospital, Osaka, Japan, between 1997 and 2000. All patients had received a mastectomy or conservative breast surgery with axillary lymph node dissection. All of the women who had undergone conservative breast surgery received postoperative radiotherapy to the residual ipsilateral breast. Each patient was treated with suitable adjuvant therapy (chemotherapy or endocrine therapy or none) postoperatively according to the stage of the disease. The characteristics of the patients are

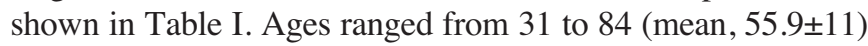
years. Forty-two patients $(31.8 \%)$ were under 50 years old; the tumors were 6-118 (average, 29.6 \pm 16.8 ) $\mathrm{mm}$ in diameter; and 69 patients $(52.3 \%)$ had lymph-node metastasis at the time of surgery. One hundred and twenty-five cases were invasive ductal carcinoma, 6 cases were invasive lobular carcinoma, and 1 case was medullary carcinoma. Noninvasive ductal, mucinous and apocrine carcinomas were excluded from this study. Fifty-two patients (39.4\%) had relapsed disease by the time of the last follow-up. Thirty patients $(22.7 \%)$ died of breast cancer. The overall 5-year survival and disease-free survival rates after the initial surgical treatment were $77.3 \%$ and $59.8 \%$, respectively, with a mean follow-up of $69 \pm 24$ months. Patients' characteristics and pathological information were obtained from their clinical records. Pathological features of the tumors were determined from standard H\&E-based observation by the two pathologists and recorded. Written informed consent was obtained from each patient, and the protocol was approved by the ethics committee of Osaka City University.

Immunohistochemistry. All tissues were fixed in $10 \%$ neutral buffered formalin immediately after surgical resection and embedded in paraffin using standard protocols. IHC was performed as reported previously $(6,7)$ on a single representative block from each case, including cancerous and noncancerous adjacent tissue. Tissue sections $(4 \mu \mathrm{m})$ were dewaxed, and antigen retrieval was performed by autoclaving at $121^{\circ} \mathrm{C}$ for $5 \mathrm{~min}$ in $10 \mathrm{mM}$ citrate buffer ( $\mathrm{pH} \mathrm{6.0)}$. Sections were incubated for $25 \mathrm{~min}$ in $3 \%$ hydrogen peroxide to quench endogenous tissue peroxidase. Primary monoclonal antibodies directed against lymphoepithelium and blood vessel epithelium, respectively, were D2-40 and CD34 (both prediluted, Nichirei, Tokyo, Japan). Tissue sections were incubated with each antibody for $70 \mathrm{~min}$ at room temperature. After washing in phosphate buffered saline, tissues were incubated with
Table I. Clinicopathologic characteristics of 132 patients investigated.

Variables

No. of cases

Age (years)

Median/range

$55.9 / 31-84$

$\leq 50$

$>50$

90

Tumor size (mm)

Median/range

$29.6 / 6-118$

$\leq 20$

44

$>20$

88

Lymph node status

Negative

Positive

Menopausal status

Pre

Post

ER

Negative

64

Positive

68

Nuclear grade

1

Histological classification

Papillotubular

Scirrhous

59

Solid-tubular

14

Invasive lobular

6

Medullary

horseradish peroxidase-conjugated anti-rabbit or anti-mouse Ig polymer as a second antibody (Envision kit, Dako Cytomation, Carpinteria, CA) for $30 \mathrm{~min}$ at room temperature, according to the manufacturer's instructions. After visualizing the immunoreaction with 3, 3'-diaminobenzidine, the sections were counter-stained with hematoxylin. The samples were examined by two investigators without knowledge of the clinical characteristics of the patient.

Assessment of lymphovascular invasion. Positive immunostaining of the lymphatic endothelium with D2-40 outlining tumor emboli in the lumen of lymphatics was defined as D2-LVI ${ }^{+}$(lymphovascular invasion), and lymphatic invasion following conventional $\mathrm{H} \& \mathrm{E}$ staining was defined as $\mathrm{HE}-\mathrm{LVI}^{+}$. D2-LVI was considered evident if at least one tumor cell cluster was clearly visible inside the D2-40-stained lymphatic vessels.

Assessment of microvessel density. For each patient, five vascularized areas ('hot spots') were chosen from intratumoral 
A

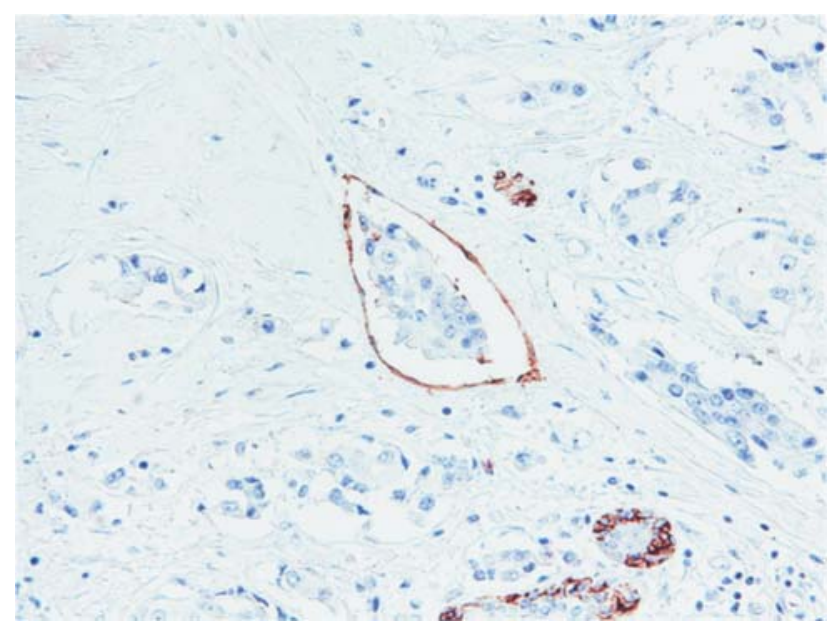

B

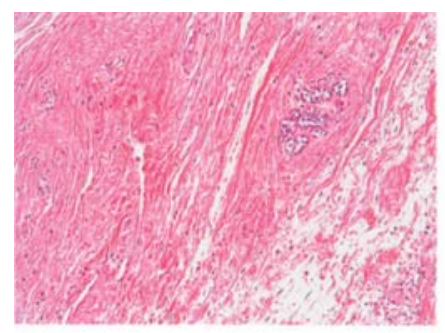

HE

C

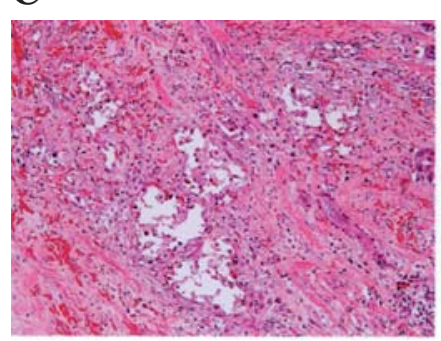

HE

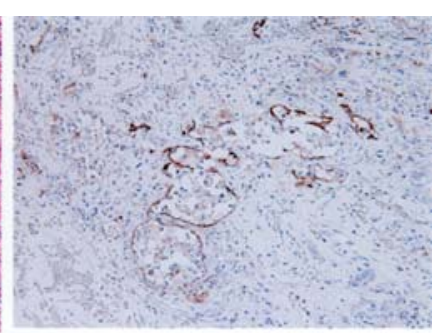

D2-40

Figure 1. Typical immunostaining patterns. A tumor embolus infiltrating the lumen of a lymphatic vessel is clearly outlined by a D2-40 positive vessel wall (A). An example of 'false positive' by H\&E staining mistaking a peritumoral or intratumoral carcinomatous vesiculous lesion for lymph duct invasion (B). An example of 'false negative' by H\&E staining, in which lymphatic invasion was shown only by D2-40 staining. Marked bending and tortuosity of lymphatic vessels interfered with accurate diagnosis by $\mathrm{H} \& \mathrm{E}(\mathrm{C})$.

positive immunostaining with CD34 at low magnification (x40). Then, vessels were counted in a representative high magnification ( $\mathrm{x} 400 ; 0.152 \mathrm{~mm}^{2} ; 0.44-\mathrm{mm}$ diameter) field in each of these five areas. Single immunoreactive endothelial cells, or endothelial cell clusters separate from other microvessels, were counted as individual microvessels. Endothelial staining in large vessels with tunica media, and nonspecific staining of nonendothelial structures were disregarded in the microvessel count. The mean visual microvessel density for CD34 was calculated as the average of the five counts. For the purpose of examining the correlation to other factors, patients were divided into two groups based on their microvessel density, those with mean counts $<40$ (MVD-L) and those with mean counts $\geq 40$ (MVD-H).
Table II. Correlation between HE-LVI and D2-LVI.

\begin{tabular}{|c|c|c|c|c|}
\hline & \multicolumn{2}{|c|}{ HE-LVI } & \multirow[b]{2}{*}{ Total } & \multirow[b]{2}{*}{$\mathrm{p}$ value } \\
\hline & - & + & & \\
\hline D2-LVI- & 51 & 26 & 77 & $<0.001$ \\
\hline D2-LVI ${ }^{+}$ & 14 & 41 & 55 & \\
\hline Total & 65 & 67 & 132 & \\
\hline
\end{tabular}

Statistical analysis. Statistical analysis was performed with the SPSS 12.0 software package (SPSS, Tokyo, Japan). Differences were considered statistically significant for $\mathrm{p}$ values $<0.05$. Correlations between the results and clinicopathological factors were analyzed by the $\chi^{2}$ test or Fisher's exact test when the assumptions of the $\chi^{2}$ test were not met. Survival curves for patients were calculated by the Kaplan-Meier method and analyzed by the log-rank test. Multivariate logistic regression analysis was performed to identify predictors of recurrence. For each variable, the odds ratio (OR) and the $95 \%$ confidence interval $(95 \% \mathrm{CI})$ were calculated.

\section{Results}

D2-LVI compared with conventional HE-LVI and blood vessels. A typical histologic picture of D2-LVI staining is shown in Fig. 1A. Here, a tumor embolus infiltrating the lumen of a dilated lymphatic vessel is clearly outlined by positive D2-40 immunostaining of the vessel wall. Intratumoral lymphatic vessels were observed extremely rarely, whereas most lymphatic vessels were located within the tumor stroma, at the front border of invasive tumor formations. Occasionally, D2-40-positive epithelial fragments that were elongated and flat were observed in the stroma between the tumor lobulations accompanied by fibrous bands. These findings were in line with observations reported previously (8-10).

HE-LVI was determined as positive in $67(50.7 \%)$ of 132 cases, whereas D2-LVI was found in 55 (41.6\%) cases. A significant correlation was observed between HE-LVI and D2-LVI ( $<<0.001)$, and results accorded in 92 samples $(69.7 \%)$ (Table II). Twenty-six (19.7\%) cases demonstrated a 'falsepositive' (HE-LVI ${ }^{+} / \mathrm{D} 2-\mathrm{LVI}^{-}$) result. In most of these cases, peritumoral or intratumoral carcinomatous vesiculous lesions had been mistaken for lymph vessel invasion, but were not lined with D2-40 positive epithelial walls (Fig. 1B). Fourteen $(10.6 \%)$ cases were determined as 'false-negative' (HE-LVI-/ $\mathrm{D} 2-\mathrm{LVI}^{+}$). In representative examples of these cases, typical lymphatic invasion was confirmed only with D2-40 staining, by demonstrating lymphovessels outlining the tumor clusters with high degrees of bending and tortuosity (Fig. 1C). Moreover, tumor cells were packed in the lymphatic vessels, and no free lumen to identify the structure was found in those specimens. In the present study, there was no case in which we misdiagnosed the micro-blood vessel invasion for lymphatic invasion with conventional $\mathrm{H} \& \mathrm{E}$ staining by confirming with CD34 staining. 
Table III. Correlation between HE-LVI/D2-LVI and clinicopathological variables.

\begin{tabular}{|c|c|c|c|c|c|c|}
\hline \multirow[b]{2}{*}{ Variables } & \multicolumn{2}{|c|}{ D2-LVI } & \multirow[b]{2}{*}{$\mathrm{p}$ value } & \multicolumn{2}{|c|}{ HE-LVI } & \multirow[b]{2}{*}{$\mathrm{p}$ value } \\
\hline & - & + & & - & + & \\
\hline \multicolumn{7}{|l|}{ Age } \\
\hline$\leq 50$ & 19 & 23 & & 16 & 26 & \\
\hline$>50$ & 58 & 32 & 0.037 & 49 & 41 & NS \\
\hline \multicolumn{7}{|l|}{ Tumor size } \\
\hline$\leq 20 \mathrm{~mm}$ & 24 & 20 & & 27 & 17 & \\
\hline$>20 \mathrm{~mm}$ & 53 & 35 & NS & 38 & 50 & 0.049 \\
\hline \multicolumn{7}{|c|}{ Menopause } \\
\hline No & 15 & 25 & & 14 & 26 & \\
\hline Yes & 62 & 30 & 0.001 & 51 & 41 & 0.031 \\
\hline \multicolumn{7}{|c|}{ Lymph node metastasis } \\
\hline Negative & 42 & 21 & & 39 & 24 & \\
\hline Positive & 35 & 34 & 0.046 & 26 & 43 & 0.005 \\
\hline \multicolumn{7}{|l|}{ ER } \\
\hline Negative & 36 & 28 & & 33 & 31 & \\
\hline Positive & 41 & 27 & NS & 32 & 36 & NS \\
\hline \multicolumn{7}{|c|}{ Nuclear grade } \\
\hline 1 & 64 & 48 & & 53 & 59 & \\
\hline $2 / 3$ & 13 & 7 & NS & 12 & 8 & NS \\
\hline \multicolumn{7}{|l|}{ Recurrence } \\
\hline No & 54 & 26 & & 42 & 38 & \\
\hline Yes & 23 & 29 & 0.008 & 23 & 29 & NS \\
\hline \multicolumn{7}{|l|}{ MVD } \\
\hline Low & 45 & 22 & & 37 & 30 & \\
\hline High & 32 & 33 & 0.037 & 28 & 37 & NS \\
\hline
\end{tabular}

Correlation of LVI and clinicopathologic variables. Each clinicopathologic variable was compared based on HE-LVI and D2-LVI by univariate analysis (Table III). Significant correlations were seen between HE-LVI and tumor size ( $\mathrm{p}=0.049)$, menopausal status $(\mathrm{p}=0.031)$, and lymph node status $(\mathrm{p}=0.005)$. Significant correlations were displayed between D2-LVI and the patient's age $(\mathrm{p}=0.037)$, menopausal status $(\mathrm{p}=0.001)$, lymph node status $(\mathrm{p}=0.046)$, disease recurrence ( $\mathrm{p}=0.008)$, and MVD ( $\mathrm{p}=0.037)$. No correlation was found between both HE-LVI and D2-LVI and estrogen receptor (ER) status or nuclear grade. When we combined the data from LVI and MVD, a strong correlation was found between positive vessel involvement and disease recurrence, as we reported previously in a preliminary setting (11). In the present study, disease recurrence was observed in $23(69.6 \%)$ of 33 cases in D2-LVI+ and MVD-H. By contrast, only 7 (15.6\%) of 45 cases showed recurrence in the D2-LVI- and MVD-L group. Based on univariate analysis, a significant correlation was demonstrated between disease recurrence and tumor size ( $\mathrm{p}<0.001)$, lymph node status $(\mathrm{p}<0.001), \mathrm{D} 2-\mathrm{LVI}(\mathrm{p}=0.008)$, and MVD $(\mathrm{p}<0.001)$ (Table IV). No correlation was found between HE-LVI and disease recurrence. On the other hand, based on multivariate logistic regression analysis, lymph node status $(\mathrm{p}<0.001, \mathrm{OR}=6.993)$, tumor size $(\mathrm{p}=0.005, \mathrm{OR}=5.504)$, D2-LVI ( $\mathrm{p}=0.006, \mathrm{OR}=4.740)$, and MVD $(\mathrm{p}=0.002$, $\mathrm{OR}=4.484$ ) remained independent prognostic factors. Still, no correlation was found between HE-LVI and disease recurrence. Once again, a significant difference in diseasefree survival was found between patients with or without D2-LVI ( $p=0.0067$, log-rank test, Fig. 2A). By contrast, no correlation was found between patients with or without HE-LVI (Fig. 2B). Among node-positive cases, a significantly higher recurrence rate was demonstrated in cases with D2-LVI than in those without D2-LVI (Fig. 3A). This significance was not demonstrated when cases were divided according to HE-LVI (Fig. 3B).

\section{Discussion}

Lymphatic vessel invasion was newly recognized as one of the factors upon which to base treatment plan decisions at the 2005 St. Gallen consensus meeting (1). Lymph-node metastasis 
Table IV. Correlation between disease recurrence and clinicopathological variables.

\begin{tabular}{|c|c|c|c|c|c|c|}
\hline \multirow[b]{2}{*}{ Variable } & \multicolumn{3}{|c|}{ Univariate analysis } & \multicolumn{3}{|c|}{ Multivariate analysis } \\
\hline & No recurrence & Recurrence & $\mathrm{p}$ value & Odds ratio & $95 \%$ CI & $\mathrm{p}$ value \\
\hline \multicolumn{7}{|l|}{ Age } \\
\hline$\leq 50$ & 25 & 17 & & 1.344 & $0.212-8.522$ & NS \\
\hline$>50$ & 55 & 35 & NS & & & \\
\hline \multicolumn{7}{|l|}{ Tumor size } \\
\hline$\leq 20 \mathrm{~mm}$ & 37 & 7 & & 5.504 & $1.682-18.009$ & 0.005 \\
\hline$>20 \mathrm{~mm}$ & 43 & 45 & $<0.001$ & & & \\
\hline \multicolumn{7}{|l|}{ Menopause } \\
\hline No & 24 & 16 & & 0.444 & $0.065-3.037$ & NS \\
\hline Yes & 56 & 36 & NS & & & \\
\hline \multicolumn{7}{|c|}{ Lymph node metastasis } \\
\hline Negative & 53 & 10 & & 6.993 & $0.052-0.393$ & $<0.001$ \\
\hline Positive & 27 & 42 & $<0.001$ & & & \\
\hline \multicolumn{7}{|l|}{ ER } \\
\hline Negative & 37 & 27 & & 1.402 & $0.522-3.762$ & NS \\
\hline Positive & 43 & 25 & NS & & & \\
\hline \multicolumn{7}{|c|}{ Nuclear grade } \\
\hline 1 & 71 & 41 & & 2.141 & $0.582-7.879$ & NS \\
\hline $2 / 3$ & 9 & 11 & NS & & & \\
\hline \multicolumn{7}{|l|}{ HE-LVI } \\
\hline - & 42 & 23 & & 2.493 & $0.137-1.177$ & NS \\
\hline+ & 38 & 29 & NS & & & \\
\hline \multicolumn{7}{|l|}{ D2-LVI } \\
\hline- & 54 & 23 & & 4.740 & $1.553-14.468$ & 0.006 \\
\hline+ & 26 & 29 & 0.008 & & & \\
\hline \multicolumn{7}{|l|}{ MVD } \\
\hline Low & 54 & 13 & & 4.484 & $0.085-0.589$ & 0.002 \\
\hline High & 26 & 39 & $<0.001$ & & & \\
\hline
\end{tabular}

A

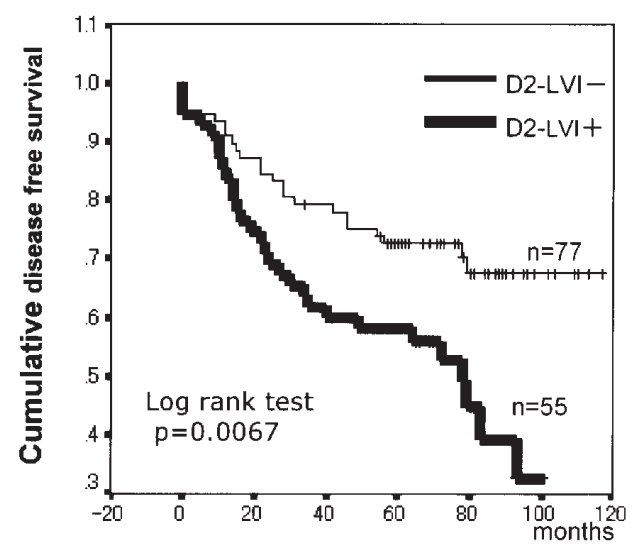

B

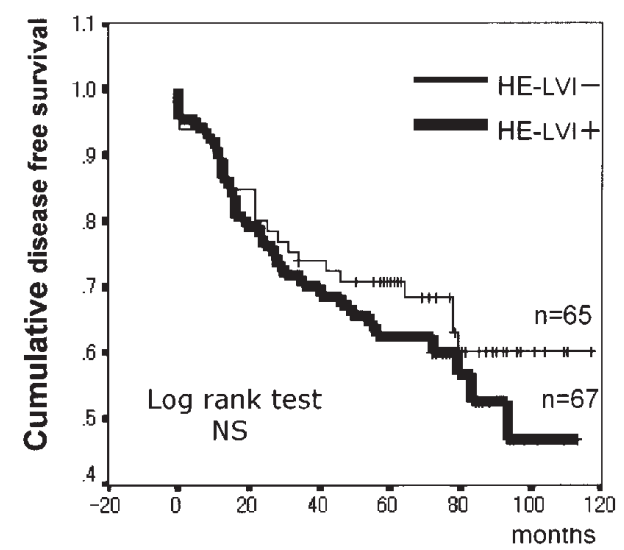

Figure 2. Kaplan-Meier survival analysis of DFS depending on D2-LVI (A) and HE-LVI (B) status. D2-LVI+ status exhibited significantly worse DFS compared with D2-LVI- ( $\mathrm{p}=0.0067, \log$-rank test), but HE-LVI status could not predict differences in DFS with statistically significant accuracy. 
A

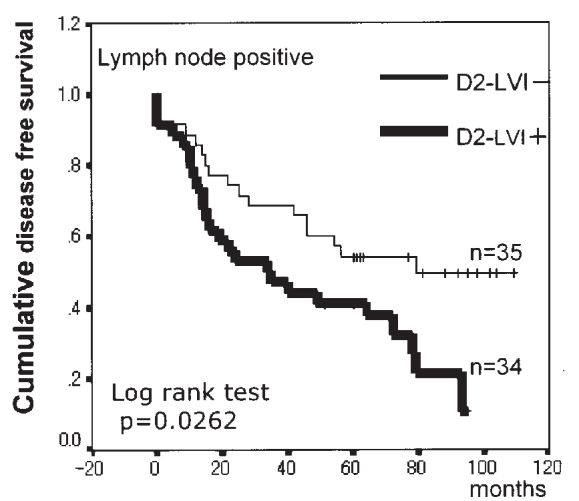

B

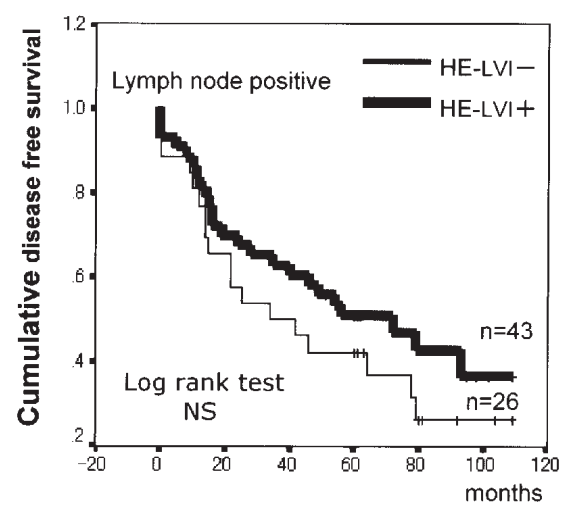

Figure 3. Kaplan-Meier survival analysis of DFS depending on D2-LVI (A) and HE-LVI (B) status in node-positive cases. D2-LVI ${ }^{+}$status exhibited significantly worse DFS compared with D2-LVI- ( $\mathrm{p}=0.0262, \log$-rank test). HE-LVI status could not predict differences in DFS with statistically significant accuracy.

of cancer cells is considered to occur by several steps including primary tumor growth, motility and invasion toward lymphatic capillaries, invasion into lymphatic lumen, embolism, entry into sentinel lymph nodes, and proliferation in the nodes (12-14). Thus, it is quite reasonable to list LVI as a therapeutic decision-making tool signaling that the tumor has already passed the initial step of lymph-node metastasis, the strongest prognostic indicator for breast cancer. Although LVI has been known to have these clinical implications, it has been also documented that pathologists can vary considerably in evaluating vessel involvement of cancer cells (3). The difference in evaluation may be influenced by many factors including examiner's prejudice caused by a global image of a tumor (15). Recently, D2-40 and other specific markers were reported to detect lymphatic epithelium specifically (3-5). Still, there have been only limited reports investigating LVI with these specific markers in breast cancer. Furthermore, no study has ever investigated the clinical significance including LVI-based prognosis in breast cancer using specific markers in comparison with that using conventional H\&E stain.

The majority of former reports have failed to display aggressive lymphangiogenesis within the tumor, and have rather demonstrated that lymphatic vessels were predominantly found peripherally or outside of the tumor or in the stroma within the tumor $(9,16-18)$. Although experimental studies revealed that lymphangiogenesis could be induced by forced expression of lymphangiogenic cytokines $(9,16,17)$, a lack of lymphangiogenesis has been displayed in clinical breast cancer specimens $(16,19)$. Further investigation is necessary to merge these results. Similarly, former investigations have revealed that LVI is seen only extremely rarely in the intratumoral area $(9,20)$. The manners of lymphatic vessel staining in breast cancer were, thus, identical in each report and the results were corroborated in this study. It should be reasonable to evaluate LVI in the tumor peripherally or in the peritumoral area.

In the present study, we detected LVI in $42 \%$ of the cases, and that rate was almost in the same range as that observed by H\&E stain $(51 \%)$. LVI detected with IHC using specific markers ranged from $28.1 \%$ (9) to $69.5 \%$ (17) in the former reports. Thus, the rate of LVI in breast cancer was not high even when we used a highly specific method. Still, as shown in this study, there was considerable discrepancy in determining LVI between H\&E and IHC. In 30\% of the cases, we found split results. Van den Eynden has also observed that $25 \%$ of the LVI could not be detected by H\&E stain (20). Not only unawareness of the lymphatic epithelium, but also overdiagnosis was found in the present study. In most occasions of misdiagnosis, the vessel was filled with tumor cells and the vessel was extraordinary elongated or compressed. It was very difficult to identify the lymphatic epithelium in these cases by H\&E stain. Therefore, IHC with a specific marker clearly enables more accurate diagnosis.

In line with the observations of others $(9,18)$, a significant correlation between LVI and lymph-node metastasis was observed in our study. On the other hand, we found many different characteristics when we classified patients according to D2-LVI rather than HE-LVI. We have demonstrated that D2-LVI correlates with younger age, premenopausal status, and MVD, but not with tumor size, ER status or nuclear grade. These clinical characteristics were quite similar with the observation of Schopmann et al, who reported the characteristics of podoplanin-positive LVI in 374 breast cancer cases (9). These results clearly indicated that the influence of the global image of the tumor was naturally eliminated by investigating LVI with a specific marker, and the real clinical features of LVI-positive cancer seemed to be revealed. Breast cancer in young pre-menopausal women is known to have poorer outcome than that in post-menopausal women. Differences in systemic hormonal circumstances have been used to explain the difference in outcomes (21). Our study might give another explanation that aggressive LVI in premenopausal women entails a poorer outcome of the disease. The mammary gland surrounding the tumor is much thicker and denser in pre-menopausal than postmenopausal women. Along with the progressive atrophy of the mammary gland after menopause, lymphatic vessels within the tissues gradually disappear. As stated earlier, lymphatic vessels in cancer predisposed breast do not seem to be generated by the tumor, are not found in the tumor but rather around it, and are usually compressed. Thus, premenopausal breasts without atrophy should have many more 
lymphatic vessels and have more chance to develop LVI in comparison with post-menopausal breasts.

Furthermore, in the present study, D2-LVI remained an independent prognostic factor to indicate disease recurrence, following nodal status and tumor size, the two most important prognostic factors in breast cancer $(8,9)$. Survival curves demonstrated that D2-LVI was significantly associated with DFS, but HE-LVI was not. In addition, HE-LVI had no meaning when node-positive patients were selected. By contrast, even in node-positive cases, D2-LVI had prognostic meaning. These results clearly demonstrated that LVI could be considered an important predictor for disease recurrence, much more than we had expected, especially when it is more specifically diagnosed with IHC, compared to H\&E stain.

In conclusion, LVI may play a crucial role in progression and disease recurrence in breast cancer. Our data revealed that D2-LVI in tumors could predict a poor disease-free survival more accurately than HE-LVI or MVD. The procedure of IHC has already been standardized and used as a routine clinical diagnosis in breast cancer to evaluate ER, progesterone receptor and Her2/neu expression. Evaluation of D2-LVI could be added to those routine procedures very easily. It is obvious that IHC by D2-40 has higher specificity for distinguishing lymphatic vessels and could help to diagnose lymphatic invasion more easily and objectively, when applied in conjunction with the established manner of standard evaluation. We propose that an evaluation of tumor peripheral lymphatic vessels only, and not intratumoral lymphatic vessels, is necessary before drawing a conclusion about the clinical significance of LVI determination.

\section{Acknowledgments}

We thank Mr. Kazuto Miyazaki for his expert technical assistance.

\section{References}

1. Goldhirch A, Glich JH, Gelber RD, et al: Meeting highlights: International Expert Consensus on the Primary Therapy of Early Breast Cancer 2005. Ann Oncol 16: 1569-1583, 2005.

2. Weidner N: Current pathologic methods for measuring intratumoral microvessel density within breast carcinoma and other solid tumors. Breast Cancer Res Treat 36: 169-180, 1995.

3. Kahn HJ and Marks A: A new monoclonal antibody, D2-40, for detection of lymphatic invasion in primary tumors. Lab Invest 82: 1255-1257, 2002.

4. Kahn HJ, Bailey P and Marks A: Monoclonal antibody D2-40, a new marker of lymphatic endothelium, reacts with Kaposi's sarcoma and a subset of angiosarcomas. Mod Pathol 15: 434-440, 2002.
5. Evangelou E, Kyzas PA and Trikalinos T: Comparison of the diagnostic accuracy of lymphatic endothelium markers: Bayesian approach. Mod Pathol 18: 1490-1497, 2005.

6. Takashima T, Onoda N, Ishikawa T, et al: Proliferating cell nuclear antigen labeling index and p53 expression predict outcome for breast cancer patients with four or more lymph node metastasis. Int J Mol Med 8: 159-163, 2001.

7. Takashima T, Onoda N, Ishikawa T, et al: Prognostic value of combined analysis of estrogen receptor status and cellular proliferative activity in breast cancer patients with extensive lymph node metastasis. Oncol Rep 9: 589-594, 2002.

8. Schoppmann SF, Birner P, Studer P, et al: Lymphatic microvessel density and lymphovascular invasion assessed by anti-podoplanin immunostaining in human breast cancer. Anticancer Res 21: 2351-2355, 2001.

9. Schoppmann SF, Bayer G, Aumayr K, et al: Prognostic value of lymphangiogenesis and lymphovascular invasion in invasive breast cancer. Ann Surg 240: 306-312, 2004.

10. Nakamura Y, Yasuoka H, Tsujimoto $\mathrm{M}$, et al: Lymph vessel density correlates with nodal status, VEGF-C expression, and prognosis in breast cancer. Breast Cancer Res Treat 91: 125-132, 2005.

11. Tezuka K, Onoda N, Takashima T, et al: Lymphatic vessel infiltration in combination with micro vessel density predicts breast cancer patients - Preliminary study with immunohistochemistry: J Clin Exp Med 217: 1139-1140, 2006 (In Japanese).

12. Cunnick GH, Jiang WG, Gomez KF and Mansel RE: Lymphangiogenesis and breast cancer metastasis. Histol Histopathol 17: 863-870, 2002.

13. Nathanson SD: Insights into the mechanisms of lymph node metastasis. Cancer 98: 413-423, 2003.

14. Thames HD, Buchholz TA and Smith CD: Frequency of first metastatic events in breast cancer: implication for sequencing of systemic and local-regional treatment. J Clin Oncol 17: 2649-2658, 1999.

15. Yamamoto H, Takiguchi Y, Kohri S, et al: Examination of lymph vessel invasion of the breast carcinoma using lymphatic endothelial marker (D2-40). Jpn J Med Tech 54: 1327-1331, 2005 (In Japanese with English abs).

16. Williams CS, Leek RD, Robson AM, et al: Absence of lymphangiogenesis and intratumoral lymph vessels in human metastatic breast cancer. J Pathol 200: 195-206, 2003.

17. Vleugel MM, Bos R, Van der Groep P, et al: Lack of lymphangiogenesis during breast carcinogenesis. J Clin Pathol 57: 746-751, 2004.

18. Kato T, Prevo R, Steers G, et al: A quantitative analysis of lymphatic vessels in human breast cancer, based on LYVE-1 immunoreactivity. Br J Cancer 93: 1168-1174, 2005.

19. Agarwal B, Saxena R, Morimiya A, et al: Lymphangiogenesis does not occur in breast cancer. Am J Surg Pathol 29: 1449-1455, 2005.

20. Van den Eynden GG, Van der Auwera I, Van Laere SJ, et al: Distinguishing blood and lymph vessel invasion in breast cancer: a prospective immunohistochemical study. Br J Cancer 94: 1643-1649, 2006

21. Pasqualini JR, Chetrite G, Blacker C, et al: Concentrations of estrone, estradiol, and estrone sulfate and evaluation of sulfatase and aromatase activities in pre- and postmenopausal breast cancer patients. J Clin Endocrinol Metab 81: 1460-1464, 1996. 